\section{Comparative analysis of cardiac cycle length during exercise in healthy people and cardiac patients}

Arterio-venous oxygen difference is very high at rest (70-80\%) and is the main determinant to set an increase in coronary blood flow as the major mechanism to meet myocardial oxygen requirements during exercise. Coronary blood flow is not evenly distributed throughout the myocardium during cardiac cycle: it is higher in the subepicardium during systole and higher in the subendocardium during diastole ${ }^{1}$ and mainly depends on two factors: heart rate (HR) and blood pressure.

The increase in coronary blood flow during exercise is produced by the increment of HR, as the increased contractility entails an increase of the compression of intramural vessels, while an increment of myocardial relaxation occurs during diastole. ${ }^{2}$ However, the increased HR produces a decrease of cardiac cycle times, specifically diastole time, resulting in a reduction of blood flow to the subendocardium. All this has led to think about the existence of a subcritical coronary stenosis, which can result in a selective subendocardial hypoperfusion during exercise. Therefore, diastolic time is considered a major determinant of oxygen delivery to myocardium. ${ }^{1}$

Our main objective was to study the variation in the duration of cardiac cycle phases in two different groups: healthy people and patients with exercise-induced cardiac ischemia. As diastole is the cardiac cycle phase where a larger increase in coronary blood flow occurs, we hypothesized that the behavior of variations of diastolic time was different between patients and healthy subjects.

Twenty three healthy asymptomatic males, and twenty four sedentary male subjects who had an exerciseinduced ischemia when undergoing a stress test. The study was approved by the local ethical committee of the Polytechnic University of Madrid, complying with the Declaration of Helsinki. All subjects were informed in detail about the tests they were going to undergo, and gave written consent to have them done. Healthy subjects were screened obtaining a 12 lead electrocardiogram (EKG) at rest, and underwent an incremental (increasing 5 watts every 12 seconds) ergoespirometry test on cycle-ergometer with real time HR and 12 lead EKG continuous monitoring. The group of patients, who had previously shown positive EKG signs of exercise-induced ischemia following a Bruce protocol, underwent a second stress test based on nuclear imaging procedures with radioisotopes.

TABLE I.-Duration of the diastolic and systolic times, in both patients and athletes, highlighting the different heart rate ranges that were analyzed.

\begin{tabular}{|c|c|c|c|c|c|c|c|c|c|}
\hline & \multicolumn{9}{|c|}{ Group } \\
\hline & \multicolumn{3}{|c|}{ Healthy } & \multicolumn{3}{|c|}{ Patients } & \multicolumn{3}{|c|}{ Total } \\
\hline & Mean & SD & N. & Mean & $\mathrm{SD}$ & $\mathrm{N}$. & Mean & SD & $\mathrm{N}$. \\
\hline D40a49 & 0.898 & 0.105 & 5 & & & & 0.898 & 0.105 & 5 \\
\hline D50a59 & 0.710 & 0.064 & 12 & 0.710 & 0.062 & 4 & 0.710 & 0.061 & 16 \\
\hline D60a69 & 0.581 & 0.043 & 18 & 0.588 & 0.031 & 5 & 0.583 & 0.040 & 23 \\
\hline D90a99 & 0.315 & 0.033 & 21 & 0.336 & 0.035 & 15 & 0.324 & 0.035 & 36 \\
\hline D100al09 & 0.267 & 0.033 & 21 & 0.293 & 0.036 & 15 & 0.278 & 0.036 & 36 \\
\hline D110all9 & 0.247 & 0.026 & 21 & 0.256 & 0.039 & 14 & 0.250 & 0.032 & 35 \\
\hline D120al29 & 0.220 & 0.020 & 21 & 0.224 & 0.032 & 11 & 0.221 & 0.024 & 32 \\
\hline D130a139 & 0.195 & 0.015 & 21 & 0.196 & 0.030 & 10 & 0.195 & 0.020 & 31 \\
\hline D170al79 & 0.138 & 0.010 & 21 & & & & 0.138 & 0.010 & 21 \\
\hline D180al89 & 0.128 & 0.011 & 21 & & & & 0.128 & 0.011 & 21 \\
\hline D190a199 & 0.123 & 0.007 & 8 & & & & 0.123 & 0.007 & 8 \\
\hline D200a209 & 0.112 & 0.008 & 5 & & & & 0.112 & 0.008 & 5 \\
\hline
\end{tabular}


TABLE II.-Duration of the diastolic and systolic times, in both patients and athletes, highlighting the different heart rate ranges that were analyzed.

\begin{tabular}{|c|c|c|c|c|c|c|c|c|c|}
\hline & \multicolumn{9}{|c|}{ Group } \\
\hline & \multicolumn{3}{|c|}{ Healthy } & \multicolumn{3}{|c|}{ Patients } & \multicolumn{3}{|c|}{ Total } \\
\hline & Mean & $\mathrm{SD}$ & $\mathrm{N}$ & Mean & $\mathrm{SD}$ & $\mathrm{N}$ & Mean & $\mathrm{SD}$ & $\mathrm{N}$. \\
\hline S40a49 & 0.40 & 0.03 & 5 & & & & 0.40 & 0.03 & 5.00 \\
\hline S50a59 & 0.38 & 0.03 & 12 & 0.36 & 0.03 & 4 & 0.38 & 0.03 & 16.00 \\
\hline S60a69 & 0.37 & 0.02 & 18 & 0.36 & 0.02 & 6 & 0.37 & 0.02 & 24.00 \\
\hline S70a79 & 0.36 & 0.03 & 20 & 0.33 & 0.02 & 12 & 0.35 & 0.03 & 32.00 \\
\hline S80a89 & 0.34 & 0.03 & 21 & 0.32 & 0.02 & 15 & 0.33 & 0.03 & 36.00 \\
\hline S90a99 & 0.32 & 0.03 & 21 & 0.31 & 0.02 & 15 & 0.31 & 0.03 & 36.00 \\
\hline S100al09 & 0.31 & 0.03 & 21 & 0.29 & 0.01 & 15 & 0.30 & 0.02 & 36.00 \\
\hline S110all9 & 0.29 & 0.02 & 21 & 0.28 & 0.01 & 14 & 0.29 & 0.02 & 35.00 \\
\hline S120al29 & 0.28 & 0.02 & 21 & 0.27 & 0.02 & 10 & 0.27 & 0.02 & 31.00 \\
\hline S130al39 & 0.26 & 0.01 & 21 & 0.26 & 0.02 & 10 & 0.26 & 0.02 & 31.00 \\
\hline S140al49 & 0.24 & 0.01 & 21 & 0.26 & 0.02 & 6 & 0.25 & 0.01 & 27.00 \\
\hline S150al59 & 0.23 & 0.01 & 21 & 0.25 & 0.02 & 5 & 0.23 & 0.02 & 26.00 \\
\hline S160al69 & 0.22 & 0.01 & 21 & 0.26 & 0.00 & 2 & 0.22 & 0.02 & 23.00 \\
\hline S170al79 & 0.21 & 0.01 & 20 & & & & 0.21 & 0.01 & 20.00 \\
\hline S180al89 & 0.20 & 0.01 & 21 & & & & 0.20 & 0.01 & 21.00 \\
\hline S190al99 & 0.19 & 0.01 & 9 & & & & 0.19 & 0.01 & 9.00 \\
\hline S200a209 & 0.18 & 0.01 & 5 & & & & 0.18 & 0.01 & 5.00 \\
\hline
\end{tabular}

TABLE III-Duration of the diastole, in both patients and healthy.

\begin{tabular}{lccccccccc}
\hline & \multicolumn{9}{c}{ Group } \\
\cline { 2 - 11 } & Mean & SD & N. & Mean & SD & N & Mean & SD & N. \\
\cline { 2 - 10 } & 0.452 & 0.042 & 20 & 0.483 & 0.039 & 12 & 0.460 & 0.042 & 32 \\
D70a79 & 0.373 & 0.036 & 21 & 0.399 & 0.044 & 15 & 0.380 & 0.039 & 36 \\
D80a89 & 0.318 & 0.031 & 21 & 0.344 & 0.045 & 15 & 0.325 & 0.036 & 36 \\
D90999 & 0.268 & 0.033 & 21 & 0.303 & 0.043 & 15 & 0.277 & 0.039 & 36 \\
D100a109 & 0.247 & 0.027 & 21 & 0.261 & 0.041 & 14 & 0.251 & 0.031 & 35 \\
D110a119 & 0.220 & 0.020 & 21 & 0.227 & 0.026 & 11 & 0.222 & 0.022 & 36 \\
D120a129 & 0.195 & 0.015 & 21 & 0.204 & 0.020 & 10 & 0.198 & 0.017 & 36 \\
D130a139 & & & & & & & & & \\
\hline
\end{tabular}

TABLE IV_-Duration of the systole, in both patients and healthy.

\begin{tabular}{lcccccrcrr}
\hline & \multicolumn{9}{c}{ Group } \\
\cline { 2 - 11 } & \multicolumn{3}{c}{ Healthy } & \multicolumn{3}{c}{ Patients } & \multicolumn{3}{c}{ Total } \\
\cline { 2 - 11 } & Mean & SD & N. & Mean & SD & N. & Mean & SD & N. \\
\hline S70a79 & 0.358 & 0.029 & 20 & 0.334 & 0.023 & 11 & 0.349 & 0.029 & 32 \\
S80a89 & 0.339 & 0.032 & 21 & 0.319 & 0.020 & 151 & 0.332 & 0.030 & 36 \\
S90a99 & 0.319 & 0.031 & 21 & 0.305 & 0.016 & 15 & 0.314 & 0.027 & 36 \\
S100a109 & 0.306 & 0.029 & 21 & 0.293 & 0.014 & 15 & 0.302 & 0.025 & 351 \\
S110a119 & 0.294 & 0.023 & 21 & 0.278 & 0.013 & 14 & 0.288 & 0.021 & 36 \\
\hline
\end{tabular}

Duration of cardiac cycle times was measured based on EKG recordings related to mechanical events, classically described by Wiggers. ${ }^{3}$ To subject the data to inferential analysis, cardiac cycle times were selected in HR ranges between 70 and 139 beats per minute (bpm).

Table I, II show the duration of diastolic and systolic times, in both groups, highlighting the different HR ranges that were analyzed. Diastole time showed a much larger decrease from resting HR to maximal HR than systole, dropping to a third of resting values. Duration of diastole was significantly different at the diverse $\mathrm{HR}$ ranges $\left(\mathrm{F}_{373}=331.5 ; \mathrm{P}=0.001\right)$ (Table III). While no significant differences were found, data seem to indicate that diastolic times in the patients group are higher than those found in the healthy group. Significant differences were found for systolic times between both groups 
$\left(\mathrm{F} !_{25}=4.4, \mathrm{P}=0.044\right)$ (Table IV), with longer systolic time in the healthy one.

Our main finding was that subjects with coronary abnormalities tend to the stabilization of systole duration (Figure 1), as opposed to healthy subjects. However, the duration of diastole shows a similar response in both groups (Figure 2); still, for each HR range, patients had higher diastolic times than healthy subjects did.

A decrease in the relative duration of diastole occurred in both groups. The shortened diastolic duration means less time for filling the vessels, which can reduce subendocardial blood flow and condition the diastolic pressure time that determines the adequacy of subendocardial perfusion. In ischemic heart disease, an increase in left ventricular diastolic pressure could further reduce subendocardial perfusion. Coronary flow can modulate the increase in myocardial contractility that occurs during exercise. Therefore, during ischemia, the longer collapse of the subendocardial vessels affects the contractility of myocardium, and may reduce cardiac output.

All this might suggest that in a trained heart, in case of coronary artery disease, the relative decrease of sys-

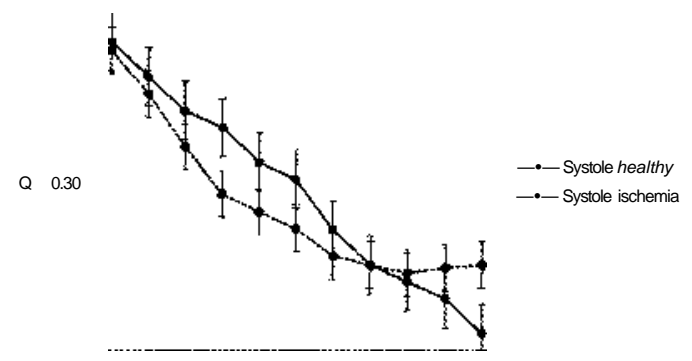

$\mathrm{HR}(\mathrm{bpm})$

Figure 1.- Systole duration in healthy people vs. cardiac patients.

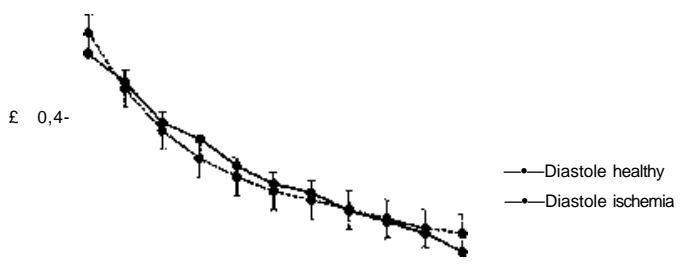

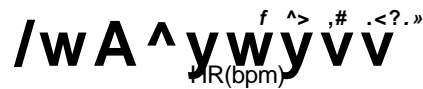

Figure 2.-Diastole duration in healthy people vs. cardiac patients. tolic time could be an advantage to a better subendocardium flow. Training may improve coronary blood flow in ischemic patients. When coronary arteries become maximally dilated, subendocardial flow is dependent upon diastolic pressure. Since trained subjects have a higher diastolic pressure during exercise, it is reasonable to assume that blood supply through both a major coronary lesion and collateral pathways would be greater in trained coronary patients. Therefore, exercise training simultaneously reduces myocardial oxygen requirements and increases potential subendocardial oxygen supply.

We detected different behaviors in systolic and diastolic times to other authors (such as Ferro et $a l^{4}{ }^{4}$ and Sugiura et al. ${ }^{5}$ who found a shortening of diastolic times in patients with ischemic electrocardiographic changes. The reasons of the disparity between our results and those of other authors could result from methodological differences or other determinants, such as the method used to determine cardiac cycle times, the data analysis strategy, the body position exercise test was performed in, or even the use of different exercise protocols.

We believe that the failure to identify significant differences in the duration of diastole can be a result of the way cardiac cycle times are defined.

During exercise, there is an increase in coronary flow as a consequence to HR increment, which leads to a decrease in the duration of cardiac cycle, thus limiting subendocardial flow. This behavior in the duration of cardiac cycle during exercise is different in healthy subjects and in patients with ischemic heart disease. Healthy subjects have a decrease in systole duration as HR increases. However, heart patients have a stabilization of systole duration from a certain HR. Therefore, in ischemic hearts, ejection capacity would be reduced at a certain exercise intensity, while ventricular in-flow would be reduced in both patients and healthy subjects.

\section{Mercedes GALINDO-CANALES \} Jesus J. ROJO-GONZALEZ I, Maria I. BARRIOPEDRO i, Juan J. RAMOS-ALVAREZ 2 *, Francisco J. CALDERON-MONTERO •

'Faculty of Sciences for Physical Activity and Sport, Madrid, Spain; ${ }^{2}$ School of Sport Medicine, Complutense University Madrid, Madrid, Spain

"Corresponding author: Juan J. Ramos-Alvarez, School of Sport Medicine, Complutense University Madrid, Plaza Ramon y Cajal s/n, 28040 Madrid. E-mail: jiramosa@ucm.es

\section{References}

1. Duncker DJ, Bache RJ. Regulation of coronary blood flow during exercise. Physiol Rev 2008;88:1009-86.

2. Brenner IK, Thomas S, Shephard RJ. Autonomic regulation of the circulation during exercise and heat exposure. Inferences from heart rate variability. Sports Med 1998;26:85-99.

3. Wiggers CJ. Studies on the consecutive phases of the cardiac cycle. Am J Physiol 1921;56:415-59. 
4. Ferro G, Guinta A, Maione S, Carella G, Genovese A, Chiariello M. Diastolic time during exercise in normal subjects and in patients with coronary artery disease. A plethysmographic study. Cardiology 1984;71:266-72.

5. Sugiura T, Iwasaka T, Takahashi N, Matsutani M, Takayama $\mathrm{Y}$, Inada M, et al. Effect of exercise on ventricular diastolic time in coronary artery disease. Am J Cardiol 1987;59:1089-92.

Conflicts of interest.- The authors certify that there is no conflict of interest with any financial organization regarding the material discussed in the manuscript.

Article first published online: September 26, 2018. - Manuscript accepted: August 28, 2018. - Manuscript revised: July 20, 2018. - Manuscript received: May 22, 2018.

(Cite this article as: Galindo-Canales M, Rojo-Gonzalez JJ, Barriopedro MI, Ramos-Alvarez JJ, Calderon-Montero FJ. Comparative analysis of cardiac cycle length during exercise in healthy people and cardiac patients. Minerva Cardioangiol 2019;67:172-5. DOI: 10.23736/S0026-4725.18.04729-1)

\section{Cardiogenic shock due to coronary fistula: a complex phenomenon}

A 48-year-old woman was admitted to our coronary care unit (CCU) due to worsening dyspnea and repeat episodes of chest pain at rest. Her past history was characterized by previous ( 20 years before) diagnosis of a congenital heart disease, which the patient decided not to treat due to personal decision in spite of recommendation to correct surgically. On admission, no documentation was available, chest pain resolved, the 12-leads electrocardiogram showed minimal STdepression in V4-V6 and cardiac enzymes were elevated. Transthoracic echocardiography revealed a large structure with internal blood flow, compressing the inferior and lateral wall of the left ventricle (Figure 1). Significant dilatation and mild contractile dysfunction of the right ventricle with severe tricuspid regurgitation and severe pulmonary hypertension were also noticed. Urgent coronary angiography showed absence of coronary atherosclerosis and a large, aneurysmatic coronary arteriovenous fistula (CAF) arising from left circumflex artery and coronary sinus, that determined compression of the proximal left anterior descending artery (LAD) (Figure 2). The right heart catheterization detected a significant left-to-right shunt due to the fistula (Qp/Qs 2). The clinical case was discussed the day after in the Heart Team and, due to the high risk, surgery was judged prohibitive. As a possible option, percutaneous revascularization was considered after detailed anatomic definition by coronary computed tomography angiography (CCTA). The CCTA scan confirmed the fistula with a diameter of $4 \mathrm{~cm}$ and an " $a b$ extrinseco" compression (estimated length: $20 \mathrm{~mm}$ ) of the proximal LAD (Figure 2). After administration of contrast material necessary for CT, determining coronary vasodilatation, another protract episode of chest pain occurred, with hemodynamic instability and a significant ST-depression in V4-V6 and II-III-aVF leads with ST-elevation in aVR (Figure 3). Therefore, an emergency coronary angiography was carried out that showed complete compression of the proximal LAD. Percutaneous coronary intervention (PCI) with drugeluting stent implantation was immediately performed (Figure 4) obtaining complete LAD lumen restoration. Yet, cardiogenic shock persisted and distal antegrade flow was not normalized. Urgent echocardiography documented significant left ventricle dysfunction, akinesia of the antero-lateral wall and severe functional mitral regurgitation. Due to the persisting hemodynam-
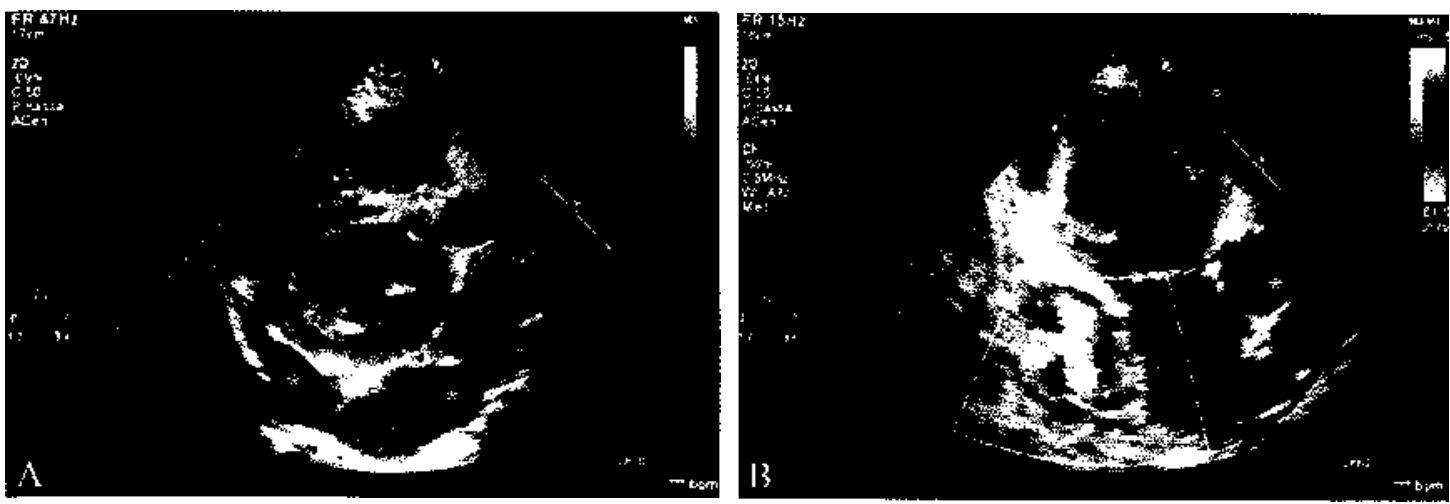

Figure 1.-Parasternal short axis view (A) and apical two-chamber view (B) at transthoracic echocardiography revealed a large structure (*), with a maximum diameter of $4 \mathrm{~cm}$ and internal blood flow, compressing the inferior and lateral wall of the left ventricle. 